\title{
Protumorigenic role of Timeless in hepatocellular carcinoma
}

\author{
NAHLA ELGOHARY ${ }^{1,2}$, ROSSELLA PELLEGRINO ${ }^{1}$, OLAF NEUMANN ${ }^{1}$, HEBA M. ELZAWAHRY ${ }^{2}$, \\ MAGDY M. SABER ${ }^{2}$, AHMED A. ZEENELDIN ${ }^{2}$, ROBERT GEFFERS ${ }^{3}$, VOLKER EHEMANN ${ }^{1}$, \\ PETER SCHEMMER $^{4}$, PETER SCHIRMACHER ${ }^{1}$ and THOMAS LONGERICH ${ }^{1}$ \\ ${ }^{1}$ Institute of Pathology, University Hospital Heidelberg, D-69120 Heidelberg, Germany; \\ ${ }^{2}$ Department of Medical Oncology (NCI), Cairo University, Cairo 11796, Egypt; \\ ${ }^{3}$ Genome Analytics, Helmholtz Centre for Infection Research, D-38124 Braunschweig; \\ ${ }^{4}$ Department of General Surgery, University Hospital Heidelberg, D-69120 Heidelberg, Germany
}

Received September 10, 2014; Accepted October 8, 2014

DOI: $10.3892 /$ ijo.2014.2751

\begin{abstract}
The mammalian timeless (TIM) protein interacts with proteins of the endogenous clock and essentially contributes to the circadian rhythm. In addition, TIM is involved in maintenance of chromosome integrity, growth control and development. Thus, we hypothesized that TIM may exert a potential protumorigenic function in human hepatocarcinogenesis. TIM was overexpressed in a subset of human HCCs both at the mRNA and the protein level. siRNA-mediated knockdown of TIM reduced cell viability due to the induction of apoptosis and G2 arrest. The latter was mediated via CHEK2
\end{abstract}

Correspondence to: Dr Thomas Longerich, Institute of Pathology, University Hospital Heidelberg, Im Neuenheimer Feld 224, D-69120 Heidelberg, Germany

E-mail: thomas.longerich@med.uni-heidelberg.de

Abbreviations: aCGH, array-based comparative genomic hybridization; ATM, ataxia telangiectasia mutated; AKT, v-akt murine thymoma viral oncogene homolog; cDNA, complementary DNA; CHEK1, checkpoint kinase 1; CHEK2, checkpoint kinase 2; CHX, cycloheximide; CSNK1E, casein kinase 1ع; Ctrl, negative control; DNA, deoxyribonucleic acid; EEF1A2, eukaryotic elongation factor 1A2; fw, forward; $\mathrm{HBV}$, hepatitis B virus; $\mathrm{HCV}$, hepatitis $\mathrm{C}$ virus; HCC, hepatocellular carcinoma; IP, immunoprecipitation; MDM2, mouse double minute homolog 2; MDM4, mouse double minute homolog 4; mTOR, mammalian target of rapamycin; NL, normal liver; OF, oligofectamine control; p21, cyclin-dependent kinase inhibitor 1A (CDKN1A, Cip1); p53, tumor protein p53; PI3K, phosphoinositide3-kinase; PAGE, polyacrylamide gel electrophoresis; PER1, period circadian clock 1; PER2, period circadian clock 2; PT, peritumorous, non-neoplastic liver tissue; PUMA, BCL2 binding component 3; qRT-PCR, quantitative real-time polymerase chain reaction; RNA, ribonucleic acid; RORA, RAR-related orphan receptor A; rev, reverse; SDS, sodium dodecyl sulfate; SEM, standard error of the mean; siNC, non-target control siRNA; siRNA, short interfering RNA; siTIM, siRNA targeting Timeless; TIM, Timeless; TIPIN, TIMELESS interacting protein; TMA, tissue microarray; WB, western immunoblotting

Key words: circadian clock genes, oncogene, hepatocellular carcinoma phosphorylation. In addition, siRNA-treated cells showed a significantly reduced migratory capacity and reduced expression levels of various proteins. Mechanistically, TIM directly interacts with the eukaryotic elongation factor 1A2 (EEF1A2), which binds to actin filaments to promote tumor cell migration. siRNA-mediated knockdown of TIM reduced EEF1A2 protein levels thereby affecting ribosomal protein biosynthesis. Thus, overexpression of TIM exerts oncogenic function in human HCCs, which is mediated via CHEK2 and EEF1A2.

\section{Introduction}

Many biological processes show a circadian rhythm, which is controlled by an endogenous clock that synchronizes with day and night phases of the solar day. The periodical rhythm is generated by a molecular oscillator located in the suprachiasmatic nuclei of the hypothalamus, which controls peripheral oscillators in virtually any other cell (1). The circadian clock is organized through a complex network of transcriptiontranslational feedback loops that drive rhythmic expression patterns of core clock components in mammals (2).

The Timeless (TIM) protein interacts with clock proteins and is essential for generation of a circadian rhythm in flies (3). In addition, phylogenetic sequence analysis revealed the presence of a paralogue in D. melanogaster (4), which is not involved in the core clock machinery, but is important for the maintenance of chromosome integrity, growth control, and development. In contrast, a single TIM gene has been identified in mammals, which acquired all of these functions as indicated by its role in DNA damage response, replication, and circadian rhythm (5-9). Consistently, TIM knockout resulted in embryonic lethality in mice (10). In addition, TIM and other clock genes have been linked to human carcinogenesis (11-14). Using integrative molecular profiling we have recently identified that inactivation of Period homolog 3 , a component of the clock machinery, occurs in human HCC indicating that dysregulation of this regulatory network may contribute to hepatocarcinogenesis (15).

The eukaryotic elongation factor $1-\alpha$ (EEF1A), a member of the $G$ protein family, represents one of the four subunits that constitute the eukaryotic elongation factor 1 (16). In humans, 
two EEF1A isoforms are known. While EEF1A1 is expressed in almost all tissues, EEF1A2 expression was only reported in heart, brain and skeletal muscle $(17,18)$. However, EEF1A2 exerts an anti-apoptotic function, which may explain its role in tumorigenesis (19). We have previously identified EEF1A2 as a candidate oncogene in human hepatocarcinogenesis and demonstrated that EEF1A2 overexpression was strongly associated with the survival probability of HCC patients $(20,21)$.

Here we analyzed the potential protumorigenic function of TIM in human hepatocarcinogenesis. Our data show that TIM overexpression promotes tumor cell proliferation via inhibition of CHEK 2 phosphorylation. In addition, TIM directly interacts with the eukaryotic elongation factor 1A2 (EEF1A2), which promotes tumor cell migration and affects ribosomal protein biosynthesis.

\section{Materials and methods}

Tumor material and patient characteristics. Expression profiles were generated from 40 human HCCs as described previously (15). The specimen included 22 liver resections and 15 explant livers; median age at surgery was 56 years (range 16-78) and the male/female ratio was 3:1. Human tissue samples were provided by the tissue bank of the National Center for Tumor Diseases Heidelberg. All diagnoses were confirmed by histological re-evaluation and use of the samples was approved by the local ethics committee. According to the vote an informed consent was not required because only long-term archived (>5 years), pseudonymized samples were used for this study. From three patients two HCC nodules were included that previously showed different aCGH data indicating independent tumor development. Etiology was determined as previously described (20). The underlying etiologies were HBV $(n=8)$, HCV $(n=8)$, alcohol $(n=7)$, cryptogenic $(n=10)$, genetic hemochromatosis $(n=3)$, and $\alpha 1$-antitrypsin deficiency $(n=1)$. The patient characteristics are shown in Table I.

Reverse transcription and polymerase chain reaction. RNA was isolated from $100 \mathrm{mg}$ of snap-frozen tissue after histological validation using the RNeasy Midi-Kit (Qiagen, Hilden, Germany) according to the manufacturer's instructions. One microgram of total RNA was reversely transcribed with the RevertAid ${ }^{\mathrm{TM}} \mathrm{H}$ minus Reverse Transcriptase (Fermentas, St. Leon-Rot, Germany) and analyzed using the ABI PRISM 7300 Real-Time PCR System (Sequence Detection software v1.2.2, Applied Biosystems, Foster City, CA, USA) with Absolute SYBR Green ROX Mix (ABgene, Epsom, UK). Calculations of efficacy, normalization, and relative quantification versus $18 \mathrm{~s}$ rRNA were done according to published algorithms (22). The following primer sequences were used (Eurofins MWG Operon, Ebersberg, Germany): TIM-fw 5'-GCC CTC AAT GTG AGG CTC TT-3', TIM-rev 5'-CCC GAA GCA GGT GAT CCT TT-3', MDM4-fw 5'-CAG CAG GTG CGC AAG GTG AA-3', MDM4-rev 5'-CTG TGC GAG AGC GAG AGT CTG-3', MDM2-fw 5'-TCT GTG AGT GAG AAC AGG TGT CAC-3', MDM2-rev 5'-ACA CAC AGA GCC AG GCT TTC-3', p21-fw 5'-CAC CGA GAC ACC ACT GGA GG-3', p21-rev 5'-GAG AAG ATC AGC CGG CGT TT-3', puma-fw 5'-AAA CGG CTA CCA CAT CCA AG-3', puma-rev 5'-CCT CCA ATG GAT CCT CGT TA-3', bax-fw
5'-TGG AGC TGC AGA GGA TGA TTG-3', bax-rev 5'-AAA CAT GTC AGC TGC CAC TCG-3', EEF1A2-fw 5'-AGA TGT CGA TGG TGA TGC-3', EEF1A2-rev 5'-AGA TGT CGA TGG TGA TGC-3', 18s-fw 5'-AAA CGG CTA CCA CAT CCA AG-3', 18s-rev 5'-CCT CCA ATG GAT CCT CGT TA-3'. Reaction temperatures and periods were used according to the manufacturer's instructions (ABgene).

DNA microarray hybridization and analysis. Quality and integrity of the total RNA was controlled using an Agilent Technologies 2100 Bioanalyzer (Agilent Technologies, Waldbronn, Germany). Total RNA (200 ng) was applied for Cy3-labelling reaction using the one color Quick Amp Labeling protocol (Agilent Technologies). Labeled cRNA was hybridized to Agilent human $8 \times 60 \mathrm{k}$ microarrays at $68^{\circ} \mathrm{C}$ for $16 \mathrm{~h}$ and scanned using the Agilent DNA Microarray Scanner (Agilent Technologies). Expression values were calculated by the software package Feature Extraction 10.5.1.1. Complete data are available online (http://www.ncbi.nlm.nih.gov/geo/ query/acc.cgi?acc=GSE50579).

Western blot analysis and immunoprecipitation. Cell samples were homogenized in lysis buffer (no. 9803, Cell Signaling Technology, Danvers, MA, USA) containing the complete protease inhibitor cocktail (Roche, Mannheim, Germany) and sonicated. Protein concentrations were determined using Nanodrop ND1000 (Thermo Scientific, Waltham, MA, USA). For Western blotting, aliquots of $100 \mu \mathrm{g}$ were denatured by boiling in Tris-glycine SDS sample buffer (Invitrogen, Karlsruhe, Germany), separated by SDS-PAGE, and blotted onto nitrocellulose membranes (Invitrogen). Membranes were blocked in 5\% non-fat dry milk in Tris-buffered saline containing $0.1 \%$ Tween-20 for $1 \mathrm{~h}$ and probed with the following specific antibodies: mouse monoclonal anti- $\beta$-actin (1:10,000; MP Biomedicals, Santa Ana, CA, USA), rabbit polyclonal anti-AKT (1:1,000; Cell Signaling Technology), mouse monoclonal anti-CHK1 (1:1,000; Cell Signaling Technology), rabbit monoclonal anti-CHK2 (1:1,000; Cell Signaling Technology), rabbit monoclonal anti-EEF1A2 (1:1,500, Abcam, Cambridge, UK), mouse monoclonal anti-MDM2 (1:200; Santa Cruz Biotechnology, Dallas, TX, USA), rabbit polyclonal anti-MDM4 (1:3,000; Abiocode, Agoura Hills, CA, USA), rabbit monoclonal anti-pAKT (Serine 473, 1:500; Cell Signaling Technology), rabbit polyclonal anti-PARP (1:500; Cell Signaling Technology), rabbit monoclonal anti-pCHK1 (Serine 345, 1:1,000; Cell Signaling Technology), rabbit polyclonal anti-pCHK2 (Threonine 68, 1:1,000; Cell Signaling Technology), rabbit polyclonal anti-TIM (1:2,000; Bethyl Laboratories, Montgomery, TX, USA), mouse monoclonal anti- $\alpha$-tubulin (1:2,000; Sigma-Aldrich, St. Louis, MO, USA). Each incubation with a primary antibody was followed by incubation with a horseradish peroxidase-conjugated secondary antibody for $1 \mathrm{~h}$ (1:2,000; Cell Signaling Technology) and visualized using the Super Signal West Pico (Pierce Chemical, New York, NY, USA). For quantification the protein densities were calculated by ImageQuaNT 5.1 software (GE Healthcare, Piscataway, NJ, USA) and normalized to $\alpha$-tubulin to determine the relative expression levels. For immunoprecipitation (IP) experiments NP40 buffer (Tris- $\mathrm{HCl} 50 \mathrm{mM}, \mathrm{NACl}$ $150 \mathrm{mM}$, NP40 1\%) was used for protein isolation. Three 
Table I. The patient characteristics of the HCC cohort used for expression profiling.

\begin{tabular}{|c|c|}
\hline \multicolumn{2}{|l|}{ Gender } \\
\hline Male & $27 \quad(73 \%)$ \\
\hline Female & $10(27 \%)$ \\
\hline Median age (range) & $56(16-78)$ \\
\hline \multicolumn{2}{|l|}{ Etiology } \\
\hline $\mathrm{HBV}$ & $8(21.6 \%)$ \\
\hline $\mathrm{HCV}$ & $8(21.6 \%)$ \\
\hline Alcohol & $7(18.9 \%)$ \\
\hline Cryptogenic & $10(27.0 \%)$ \\
\hline Genetic hemochromatosis & $3(8.1 \%)$ \\
\hline Others & $1 \quad(2.7 \%)$ \\
\hline \multicolumn{2}{|l|}{ Grading } \\
\hline Well differentiated HCC & $4(10.0 \%)$ \\
\hline Moderately differentiated HCC & $31(77.5 \%)$ \\
\hline Poorly differentiated HCC & $5(12.5 \%)$ \\
\hline \multicolumn{2}{|l|}{ Tumor size } \\
\hline$\leq 5.0 \mathrm{~cm}$ & $18(48.6 \%)$ \\
\hline$>5.0 \mathrm{~cm}$ & $19(51.4 \%)$ \\
\hline \multicolumn{2}{|l|}{ UICC stage } \\
\hline I & $24(64.9 \%)$ \\
\hline II & $11(29.7 \%)$ \\
\hline III & $0 \quad(0 \%)$ \\
\hline IV & $2(5.4 \%)$ \\
\hline \multicolumn{2}{|l|}{ Vascular invasion } \\
\hline Present & $10(27.0 \%)$ \\
\hline None & $27(73.0 \%)$ \\
\hline \multicolumn{2}{|l|}{ Liver cirrhosis } \\
\hline Present & $22(59.4 \%)$ \\
\hline
\end{tabular}

milligrams of HCC cell protein lysate were immunoprecipitated with $6 \mu \mathrm{g}$ of protein A agarose-bound EEF1A2 rabbit polyclonal antibody (Santa Cruz Biotechnology) at $4^{\circ} \mathrm{C}$ overnight. After immunoblotting, the membranes were incubated with a rabbit monoclonal anti-EEF1A2 antibody $(1: 1,500$; Abcam), rabbit polyclonal anti-TIM (Bethyl Laboratories) or mouse monoclonal anti-actin antibody (MP Biomedicals). To exclude unspecific binding IP was carried out without protein input as negative control. Respectively, mouse monoclonal anti-rabbit and goat anti-mouse light chain $\operatorname{IgG}(1: 7,000$; Jackson ImmunoResearch Laboratories, West Grove, PA, USA) were used as secondary antibodies to detect EEF1A2 bound proteins.

Tissue microarrays and immunohistochemistry. A tissue microarray (TMA) containing tissue from normal livers $(\mathrm{n}=27)$, non-tumor liver tissue of HCC patients $(n=86)$, and HCCs ( $n=102$; Table II) was constructed as previously described (23), and immunohistochemistry was performed on 5- $\mu \mathrm{m}$ sections. From eleven patients two independent HCC nodules were included. The following primary antibodies were used
Table II. The patient characteristics of TMA cohort.

\begin{tabular}{|c|c|}
\hline \multicolumn{2}{|l|}{ Gender } \\
\hline Male & $72(79 \%)$ \\
\hline Female & $19(21 \%)$ \\
\hline Median age (range) & $59(17-78)$ \\
\hline \multicolumn{2}{|l|}{ Etiology } \\
\hline $\mathrm{HBV}$ & $17(18.7 \%)$ \\
\hline $\mathrm{HCV}$ & $27(29.7 \%)$ \\
\hline Co-infection & $5 \quad(5.5 \%)$ \\
\hline Alcohol & $21(23.1 \%)$ \\
\hline Cryptogenic & $18(19.8 \%)$ \\
\hline Genetic hemochromatosis & $3(3.3 \%)$ \\
\hline \multicolumn{2}{|l|}{ Grading } \\
\hline Well differentiated HCC & $19(18.6 \%)$ \\
\hline Moderately differentiated HCC & $65(63.7 \%)$ \\
\hline Poorly differentiated HCC & $18(17.6 \%)$ \\
\hline \multicolumn{2}{|l|}{ Tumor size } \\
\hline$<2.0 \mathrm{~cm}$ & $8(8.8 \%)$ \\
\hline $2.0-5.0 \mathrm{~cm}$ & $51(56.0 \%)$ \\
\hline$>5.0 \mathrm{~cm}$ & $32(35.2 \%)$ \\
\hline \multicolumn{2}{|l|}{ UICC stage } \\
\hline I & $39(42.9 \%)$ \\
\hline II & $32(35.2 \%)$ \\
\hline III & $15(16.5 \%)$ \\
\hline IV & $5(5.5 \%)$ \\
\hline \multicolumn{2}{|l|}{ Vascular invasion } \\
\hline Present & $32(35.2 \%)$ \\
\hline None & $59(64.8 \%)$ \\
\hline \multicolumn{2}{|l|}{ Liver cirrhosis } \\
\hline Present & $58(63.7 \%)$ \\
\hline
\end{tabular}

for incubation: rabbit monoclonal EEF1A2 (1:1,500; Abcam), rabbit polyclonal Timeless (1:2,000; Bethyl Laboratories). Antigens were retrieved using citrate buffer ( $\mathrm{pH} \mathrm{6.1;} \mathrm{Dako,}$ Glostrup, Denmark). For detection the EnVision method (Dako) was used. Counterstaining was performed using hemalum. Staining was assessed using the immunoreactive score as described previously (20): 0, absent; $1-4$, weak; 5-8, moderate; 9-12, strong expression.

Cell lines, transfection and functional analyses. HepG2, Hep3B, HLE, HLF, HuH6, HuH7, PLC/PRF/5, SNU182 and SNU387 cells were cultured either in DMEM, MEM or RPMI medium, supplemented with $10 \%$ fetal bovine serum (PAA, Pasching, Austria) and $1 \%$ penicillin-streptomycin $(10 \mathrm{mg} / \mathrm{ml}, \mathrm{PAA})$ at $37^{\circ} \mathrm{C}\left(5 \% \mathrm{CO}_{2}\right)$ and passaged every 3-4 days. All siRNA transfections were performed using oligofectamine (Invitrogen) according to the manufacturer's protocol. The following small interfering RNAs were used (siRNA, Eurofins MWG Operon): siTIM1 5'-AGA AGA GAA GGA AGA AGA A-dTdT3', siTIM3: 5'-GCC UAC AUG UGC UAG AGA U-dTdT3', neutral control siRNA (siNC) 5'-UUC 


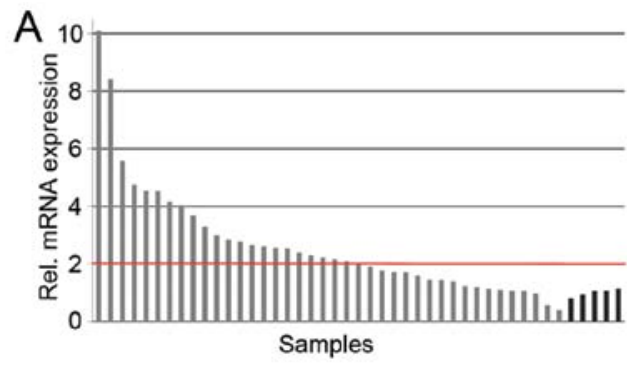

C

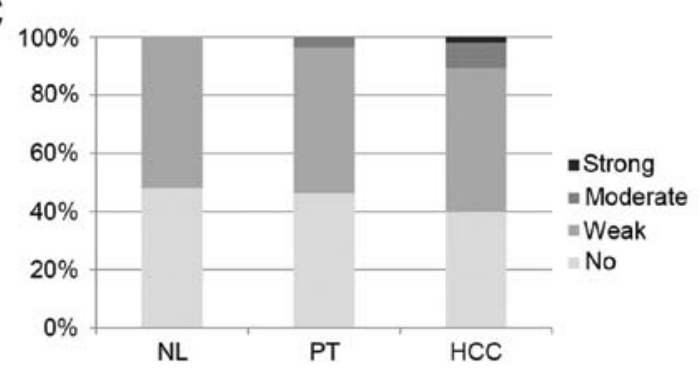

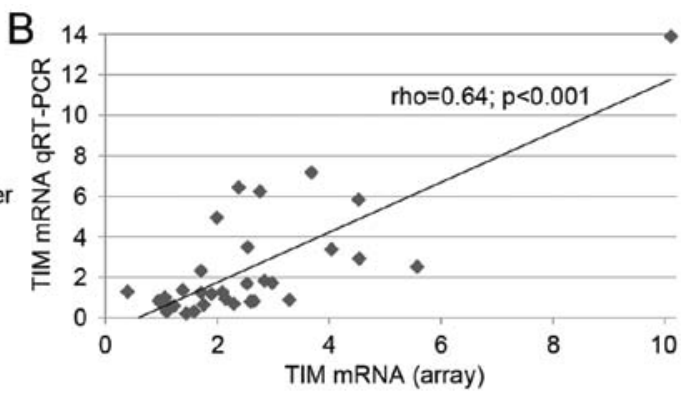

D

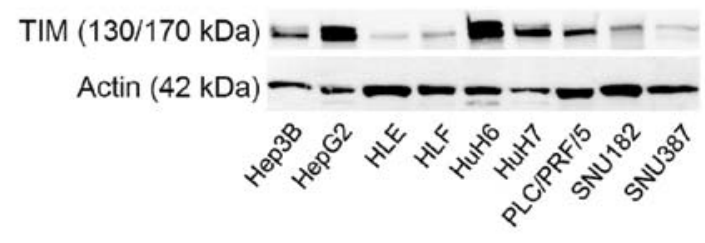

$\mathrm{E}$

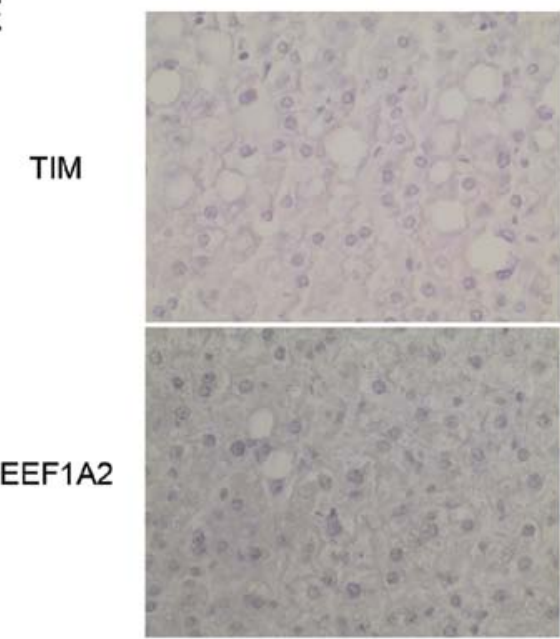

Normal liver

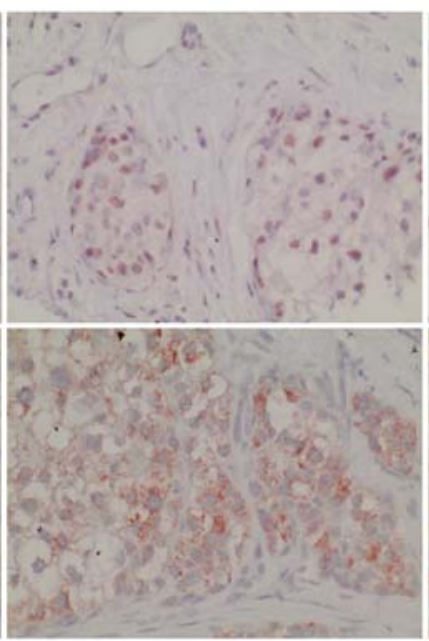

$\operatorname{HCC}(\mathrm{G} 2)$

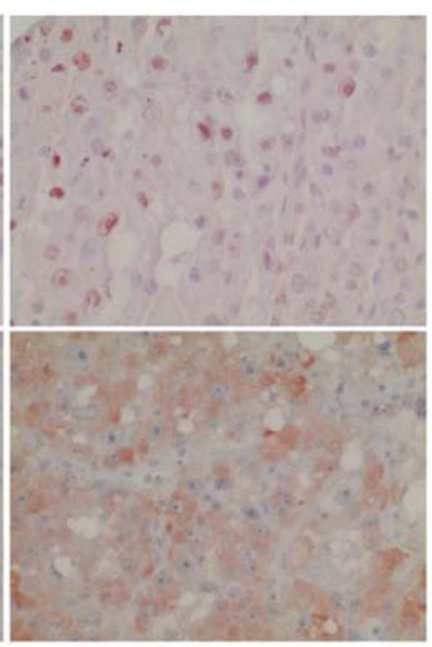

$\operatorname{HCC}(\mathrm{G} 3)$

Figure 1. TIM is upregulated in a subset of human HCCs. (A) TIM mRNA expression in human HCCs and normal liver as determined by expression profiling. (B) Good correlation of TIM mRNA expression levels between array and quantitative RT-PCR data. (C) Summary of TIM protein expression in human liver tissues using a tissue microarray [normal liver (NL), peritumorous, non-neoplastic liver tissue (PT), and HCC]. (D) TIM protein expression in various HCC cell lines. (E) Immunohistochemistry for TIM and EEF1A2 in normal liver and human HCC. Representative immunohistochemical stainings of the tissue microarray. High nuclear TIM expression is associated with EEF1A2 expression. Original magnification, x40. NL, normal liver; HCC (G2), moderately differentiated HCC; HCC (G3), poorly differentiated HCC.

UCC GAA CGU GUC ACG U-dTdT3'. Transient transfection experiments of Hep3B cells with EEF1A2 cDNA in pCMV6-XL5 vector (OriGene Technologies, Rockville, MD, USA) was performed following the manufacturer's protocol using FuGene HD transfection reagent (Promega, Mannheim, Germany). For functional assays HCC cell lines were seeded at a density of $6 \times 10^{3}$ cells in 96-well plates and were transfected with siRNA after 24 h. Cell viability (MTT-assay; M2128, Sigma, Deisenhofen, Germany), apoptosis (FACS-assay; FACS Calibur ${ }^{\mathrm{TM}}$, BD Bioscience, Heidelberg, Germany), and migration (2D-scratch-assay) were determined as described previously $(15,20)$. For the treatment with chemical inhibitors, HCC cells were plated at a density of $1.5 \times 10^{5}$ cells/well in $6-\mathrm{cm}$ plates and were incubated with the following drugs after $24 \mathrm{~h}$, as indicated: cycloheximide (protein synthesis inhibitor; Merck, Darmstadt, Germany), MG132 (proteosomal inhibitor,
$450 \mu \mathrm{mol} / 1$; Enzo Life Sciences, Lörrach, Germany). For all cell based assays, results were confirmed in three independent experiments.

Immunofluorescence microscopy. After $24 \mathrm{~h}$ of culturing on glass coverslips HCC cells were transfected using siRNAs as described above. Seventy-two hours after transfection cells were washed repeatedly in PBS containing $2 \mathrm{mM} \mathrm{MgCl}$ at $37^{\circ} \mathrm{C}$, and fixed with methanol/acetone. Fixed cells were again washed with PBS for $30 \mathrm{~min}$, permeabilized in PBS with $0.05 \%$ Tween-20 for $10 \mathrm{~min}$, and processed as described previously (24) using a mouse monoclonal $\beta$-actin antibody (1:5,000; MP Biomedicals). Fluorescence was detected using an Olympus BX40 microscope (Olympus, Hicksville, NY, USA) equipped with a Leica DFC365FX camera (Leica, Wetzlar, Germany). 

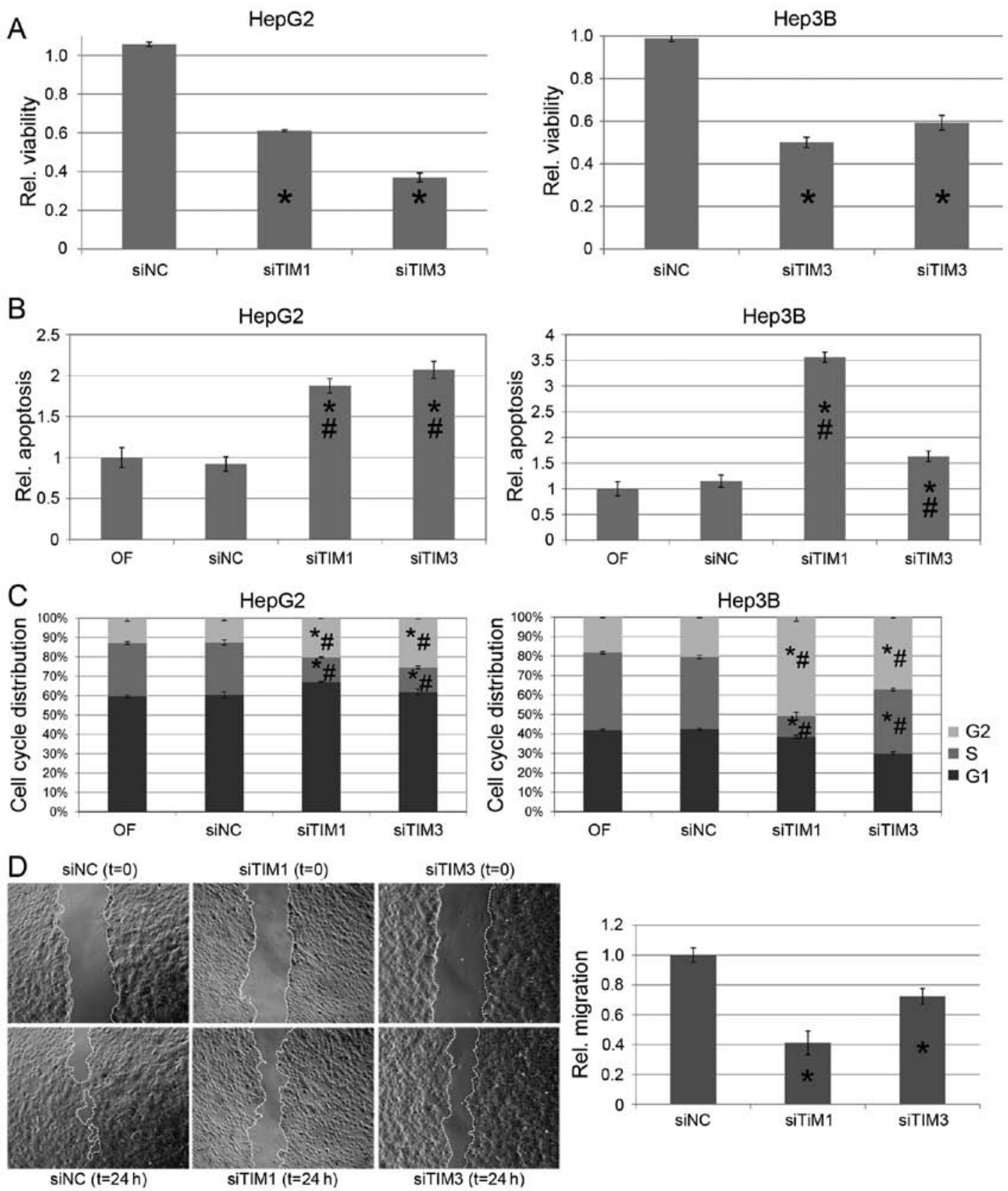

siTIM3 $(\mathrm{t}=0)$
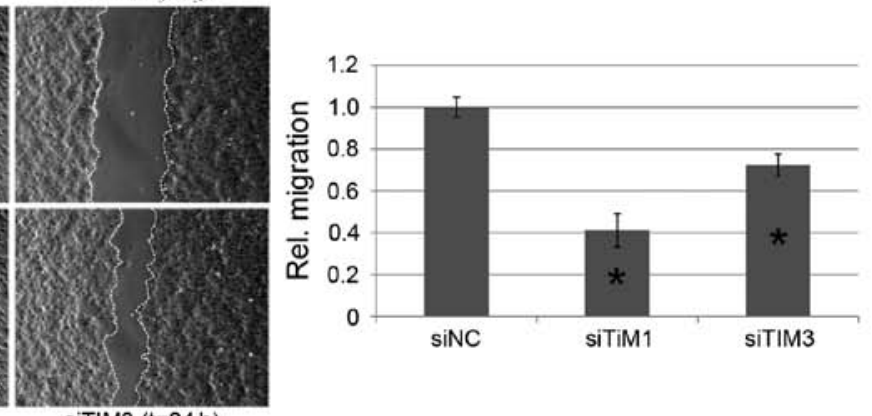

Figure 2. Functional analyses following siRNA-mediated silencing of TIM in HCC cell lines. (A) Knockdown of TIM using two independent siRNAs reduces the cell viability ( ${ }^{*}$ siNC vs. siTIM, $\left.\mathrm{P}<0.05\right)$ and $(\mathrm{B})$ induces apoptosis in HepG2 and Hep3B cells compared to shNC-treated cells, respectively ${ }^{\sharp} \mathrm{OF}$ vs. siTIM vs. $\mathrm{OF}, \mathrm{P}<0.05$; " siNC vs. siTIM, $\mathrm{P}<0.05$ ). (C) A significant $\mathrm{G} 2$ arrest is noted in both cell lines, while the fraction of cells in $\mathrm{G} 1$ is also increased in Hep3B cells ( ${ }^{\#} \mathrm{OF}$ vs. siTIM vs. $\mathrm{OF}, \mathrm{P}<0.05$; ${ }^{*}$ siNC vs. siTIM, $\mathrm{P}<0.05$ ). (D) Migration is significantly reduced in Hep3B cells following TIM knockdown ("siNC vs. siTIM, $\mathrm{P}<0.05)$.

Statistical analyses. The correlation between gene expression and clinicopathological parameters was tested by Wilcoxon-signed rank tests and measured by Spearman's rank correlations. $\mathrm{P}<0.05$ was considered statistically significant. Statistical analyses were conducted using SPSS 20.0 (SPSS, Chicago, IL, USA).

\section{Results}

TIM is upregulated in a subset of human HCCs. According to our previous expression profiling (15) upregulation of TIM mRNA ( $>2$-fold) was observed in $55 \%$ of HCCs $(n=22)$, while only 1 tumor $(2.5 \%)$ showed lower expression $(<50 \%)$ compared to normal liver (Fig. 1A). These findings were validated using quantitative real-time RT-PCR (Fig. 1B). Thus, there was a significant upregulation of TIM mRNA in human HCCs compared to normal liver $(\mathrm{P}=0.004)$. Tumors measuring $>5 \mathrm{~cm}$ in diameter showed significantly higher TIM mRNA levels compared to smaller lesions $(\mathrm{P}=0.01)$. Although there was a trend for increased TIM expression with tumor dedifferentiation, the results did not reach statistical significance (rho=0.3, $\mathrm{P}=0.07$ ). No significant association was found with gender, etiology, vascular invasion and UICC stage $(\mathrm{P}>0.05)$.

Next we determined the TIM protein expression in human HCCs using TMA (Fig. 1C and E). Weak TIM expression was seen in $52 \%(n=14)$ of normal liver tissues and the remaining 


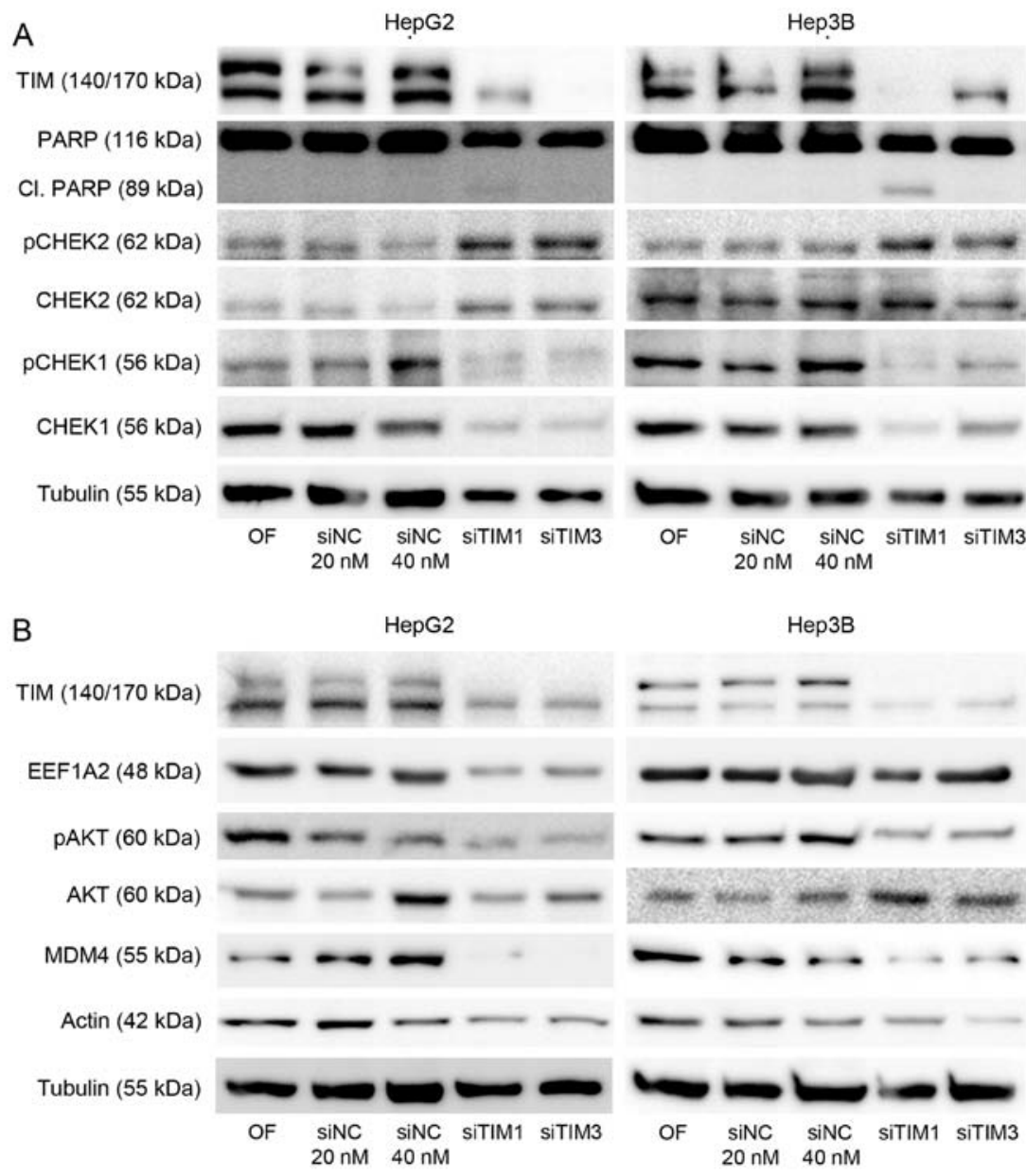

Figure 3. (A) siRNA-mediated silencing of TIM results in apoptosis in HepG2 and Hep3B cells as shown by PARP cleavage and induction of CHEK2 protein and its phosphorylation, while CHEK1 protein is reduced. (B) In addition, the protein levels of EEF1A2, AKT, MDM4, and actin as well as the phosphorylation of AKT are decreased following TIM knockdown in both cell lines.

were negative. In non-tumorous liver tissues of $\mathrm{HCC}$ patients $(\mathrm{n}=86), 47 \%$ displayed no detectable TIM signal at all, whereas half of the samples showed weak $(n=43)$ and $3 \%$ showed moderate expression of TIM. Regarding HCCs $(\mathrm{n}=103), 40 \%$ did not show any, 50\% showed weak, 9\% moderate, and $2 \%$ showed strong TIM staining. There was a trend towards higher TIM protein expression in primary HCCs that had developed extrahepatic metastasis compared to non-metastasized tumors $(\mathrm{P}=0.05)$. No significant association was found with gender, etiology, vascular invasion, and tumor size $(\mathrm{P}>0.05)$.

TIM promotes cell viability and tumor cell migration. TIM protein was expressed in all HCC cell lines analyzed. High TIM expression levels were observed in Hep3B, HepG2, HuH6, $\mathrm{HuH7}$, and PLC/PRF/5 cells (Fig. 1D). To test whether TIM exerts protumorigenic functions in vitro, we used gene-specific siRNAs to knock down TIM expression in HepG2 and Hep3B cells, which resulted in significant reduction of cell viability compared to mock transfected cells $[0.61 \pm 0.03$ (siTIM1) and $0.37 \pm 0.11$ (siTIM3) in HepG2; $0.50 \pm 0.10$ (siTIM1) and $0.59 \pm 0.17$ (siTIM3) in Hep3B cells, $\mathrm{P}<0.01$ respectively; Fig. 2A], and was associated with a mild induction of apoptosis as shown by FACS analysis [1.87 \pm 0.09 -fold (siTiM1) and 2.07 \pm 0.10 -fold (siTIM3) in HepG2 and 3.56 \pm 0.10 -fold
(siTIM1) and 1.62 \pm 0.10 -fold (siTIM3) in Hep3B compared to Mock transfected cells, each $\mathrm{P}<0.01$; Fig. $2 \mathrm{~B}]$ as well as cleavage of PARP in Western immunoblotting (Fig. 3A). In addition, knockdown of TIM resulted in a significant $\mathrm{G} 2$ arrest in both cells lines analyzed [20.3 $\pm 0.2 \%$ (siTIM1) $/ 25.8 \pm 0.5 \%$ (siTIM3) vs. $12.8 \pm 1.2 \%$ (siNC) in HepG2 and $50.5 \pm 2.1 \%$ (siTIM1) $/ 37.4 \pm 0.4 \%$ (siTIM3) vs. $20.2 \pm 0.3 \%$ in Hep3B cells, $\mathrm{P}<0.01$ respectively; Fig. 2C]. Finally, migration was significantly reduced in TIM-depleted compared to siNC-transfected Hep3B cells [0.41 \pm 0.08 (siTIM1) and $0.72 \pm 0.05$ (siTIM3), $\mathrm{P}<0.01$ respectively; Fig. 2D]. Due to their multilayer and colony forming growth characteristics the migration of HepG2 cells could not be explored in a standardized manner.

Mechanisms involved in pro-proliferative and anti-apoptotic function of TIM. As TIM has been reported to affect cell proliferation either via CHEK1- or CHEK2-mediated mechanisms $(8,25)$, we analyzed both expression and phosphorylation of these proteins. Indeed, both CHEK2 protein levels as well as CHEK2 phosphorylation were increased in HepG2 and Hep3B cells after knockdown of TIM expression (Fig. 3A). Interestingly, we observed that numerous other proteins including CHEK1 (Fig. 3A), EEF1A2, v-akt murine thymoma viral oncogene homolog (AKT), and $\beta$-actin (Fig. 3B) were downregulated following TIM knockdown suggesting that 


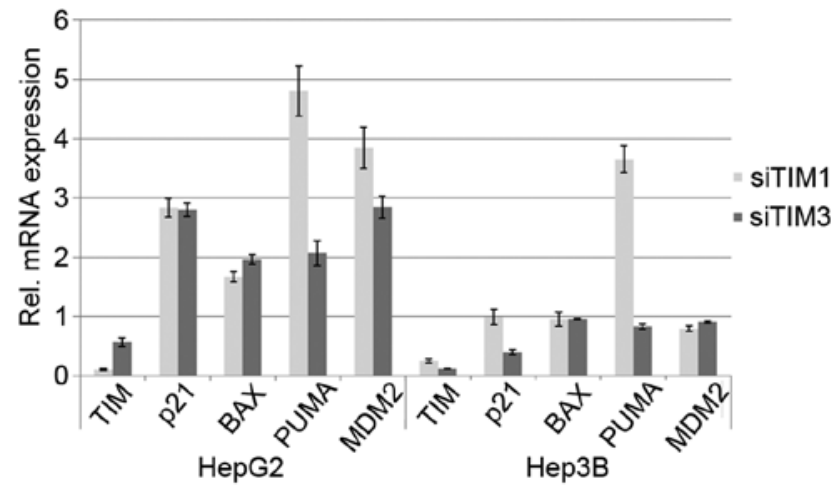

Figure 4. Rel. mRNA expression of TIM, p21, BAX, PUMA, and MDM2 in HepG2 (p53 wild-type) and Hep3B (p53-deficient) cells following siRNAmediated knockdown of TIM.

TIM may activate the protumorigenic EEF1A2/AKT/MDM4 axis (21). In line, transcriptional activation of some p53 target genes was seen following TIM knockdown in p53-wild-type cells indicating reactivation of p53 function in these cells (Fig. 4).

EEF1A2 is a crucial mediator of protumorigenic TIM functions. EEF1A2 has been shown to promote oncogenic functions in HCC (20) and was downregulated upon TIM knockdown. In addition, we observed a correlation (rho $=0.37, \mathrm{P}>0.001)$ between EEF1A2 and TIM protein as determined by immunohistochemistry (Fig. 1E). As EEF1A2 is involved in ribosomal function as well as actin dynamics, we hypothesized that EEF1A2 could be a crucial mediator of the protumorigenic properties of TIM. To explore whether EEF1A2 was able to revert the TIM-induced migratory phenotype, we transiently expressed an EEF1A2 cDNA after TIM knockdown. Both, EEF1A2 mRNA and protein levels were increased (Fig. 5A and $\mathrm{B}$ ), which was paralleled by an increase in migratory capacity compared to Mock transfected cells. However, both the migration of siTIM1-treated Hep3B cells as well as their EEF1A2 protein level remained lower compared to siNC-treated Hep3B cells (Fig. 5B and C).
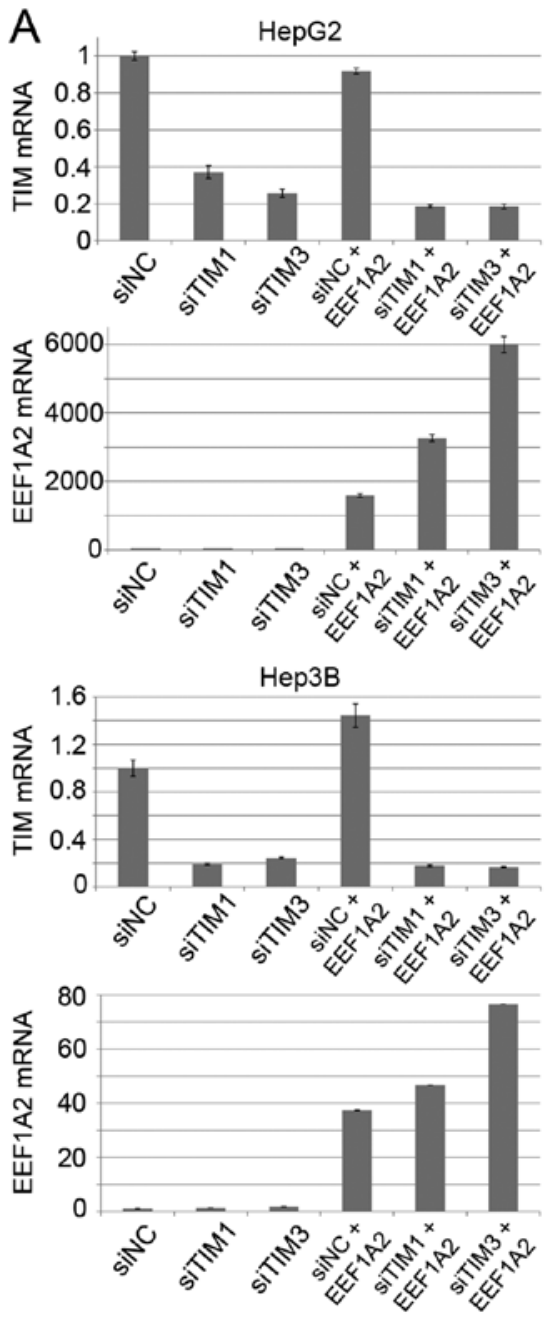

B

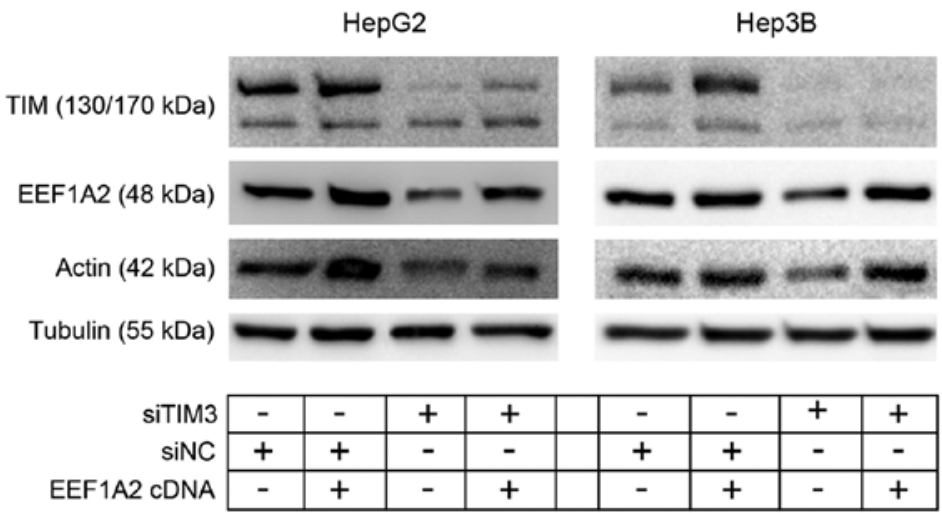

C

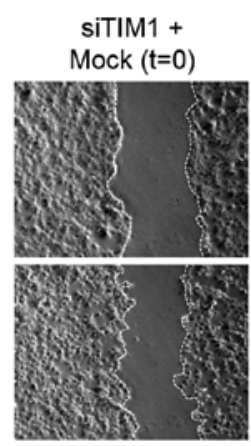

siTIM1 + $\operatorname{Mock}(\mathrm{t}=24 \mathrm{~h})$
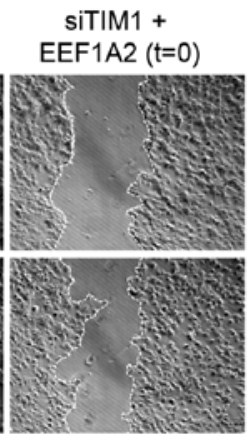

SiTIM1 +

EEF1A2 $(\mathrm{t}=24 \mathrm{~h})$

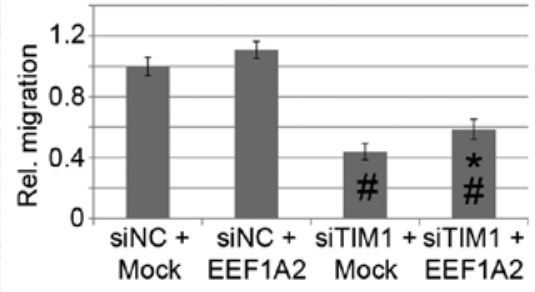

Mock EEF1A2 Mock EEF1A2

Figure 5. Transient overexpression of EEF1A2 partially rescues the migratory capacity of Hep3B cells. (A) TIM and EEF1A2 mRNA-expression following EEF1A2 cDNA expression with/without simultaneous siRNA inhibition of TIM. (B) Western immunoblotting following EEF1A2 cDNA expression with/ without simultaneous siRNA inhibition of TIM or a non-target control. (C) Migration is significantly reduced in siTIM-compared to siNC-treated Hep3B cells, but transient expression of an EEF1A2 cDNA partially rescues this phenotype. Representative microphotographs (left panel), quantification (right panel; ${ }^{*}$ siNC vs. siTIM1, $\mathrm{P}<0.05$; "Mock vs. EEF1A2 cDNA, $\mathrm{P}<0.01$ ). 
A

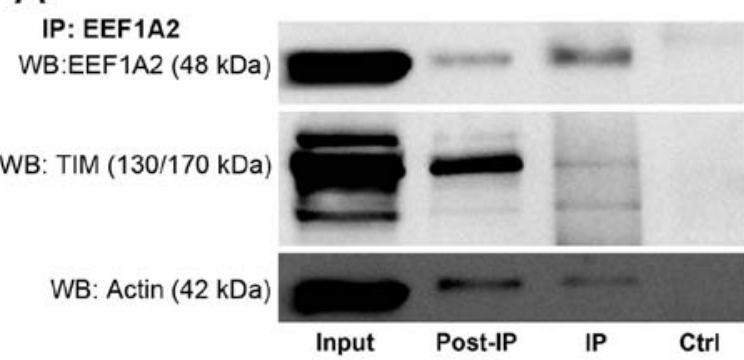

C

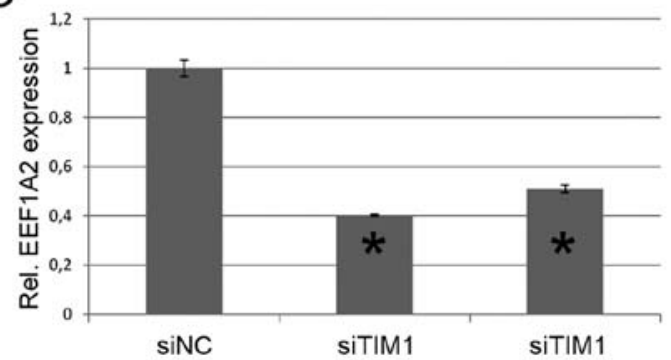

B

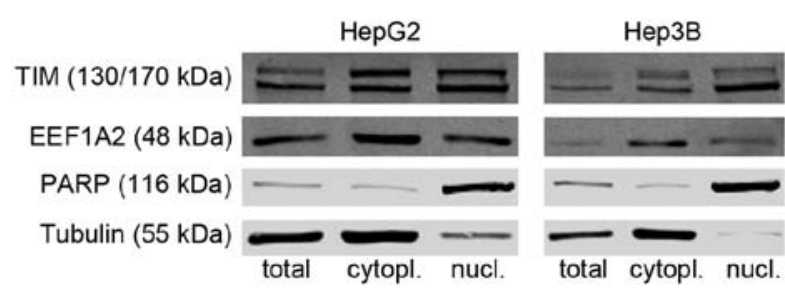

D

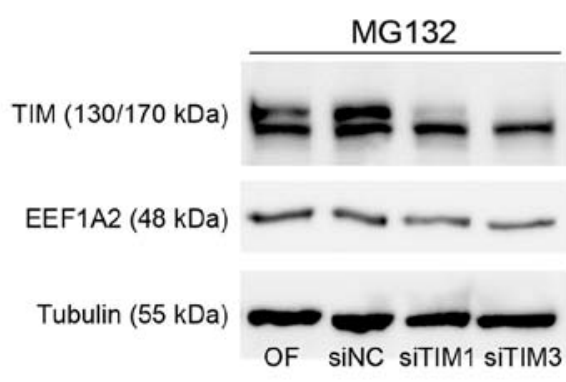

Figure 6. Protumorigenic interaction of TIM and EEF1A2. (A) Coimmunoprecipitation demonstrating a physical interaction between EEF1A2 and TIM as well as EEF1A2 and $\beta$-actin in Hep3B cells. (B) TIM and EEF1A2 can be detected in both nuclear and cytoplasmic protein extracts. (C) Densitometric analysis of EEF1A2 immunoblots following combined siRNA and and CHX treatment. TIM knockdown results in significantly lower EEF1A2 protein levels $4 \mathrm{~h}$ following inhibition of protein biosynthesis compared to siNC-treated Hep3B cells, respectively. The given values represent the EEF1A2 protein expression normalized against the $\alpha$-tubulin. (D) Inhibition of proteasomal degradation does not rescue the EEF1A2 protein levels after TIM knockdown in Hep3B cells ("siNC vs. siTIM1/siTIm3, P<0.05).
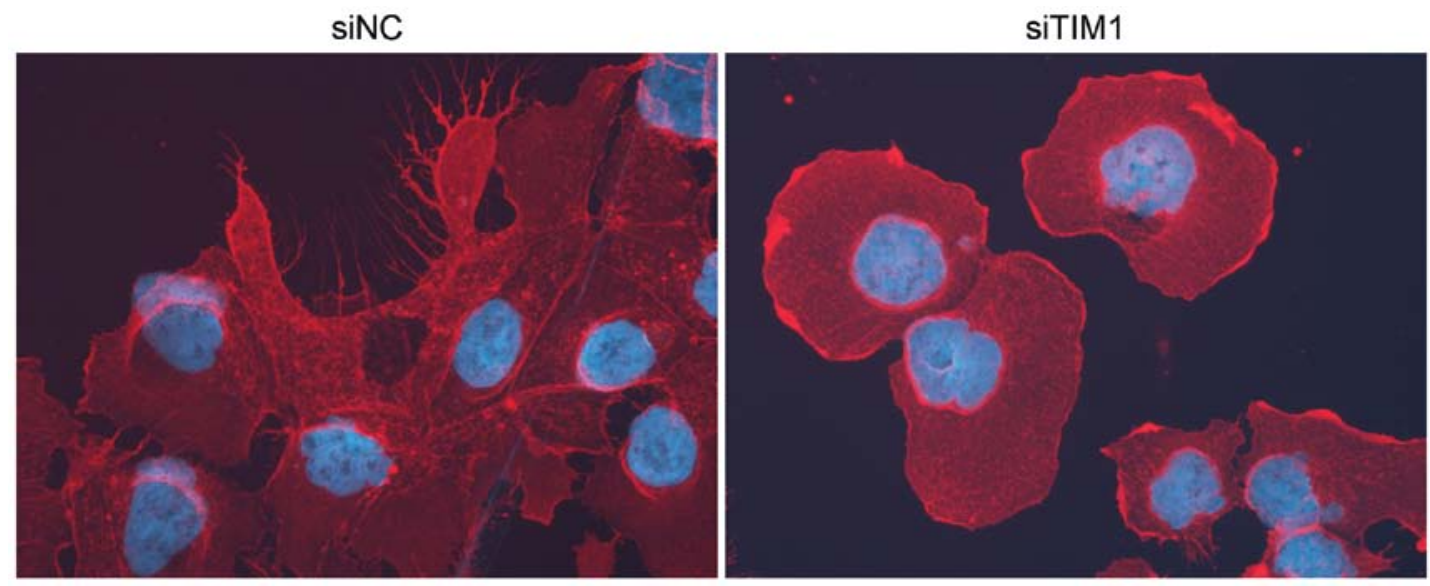

Figure 7. Filopodia formation is decreased following siRNA-mediated knockdown of TIM (actin immunofluorescence, original magnification x1,000).

Immunoprecipitation experiments revealed a direct interaction of EEF1A2 with both TIM and $\beta$-actin (Fig. 6A). In addition, filopodia formation was impaired following TIM knockdown (Fig. 7) indicating that the TIM-EEF1A2 interaction may promote actin dynamics and thus tumor cell migration. As TIM was expressed predominantly in the nucleus, while EEF1A2 was present in the cytoplasm (Fig. 1E), we further explored in which cellular compartment TIM and EEF1A2 might interact. Cellular fractionation confirmed that EEF1A2 was mainly localized in the cytoplasm, while TIM expression was observed in both compartments suggesting that the TIM-EEF1A2 interaction occurs in the cytoplasm (Fig. 6B).

Although TIM seemed to stabilize EEF1A2 via direct interaction, this finding did not explain the reduction of other proteins like CHEK1, AKT, or $\beta$-actin following TIM knockdown. In humans, two EEF1A protein isoforms (EEF1A1 and EEF1A2), which have similar elongation activity, are known $(17,18)$. Thus, we evaluated the effect of TIM knockdown on protein biosynthesis. EEF1A2 protein levels were significantly reduced after cycloheximide treatment in siTIM-compared to siNC-treated cells (Fig. 6C), while inhibition of proteasomal degradation using MG132 did not prevent reduction of EEF1A2 expression following TIM knockdown (Fig. 6D) indicating that impaired protein biosynthesis, and not increased proteasomal degradation, may be responsible for this phenotype.

Thus, our data show that TIM exerts protumorigenic functions in human HCC, which are crucially mediated by physical interaction with EEF1A2 and downstream activation of the 


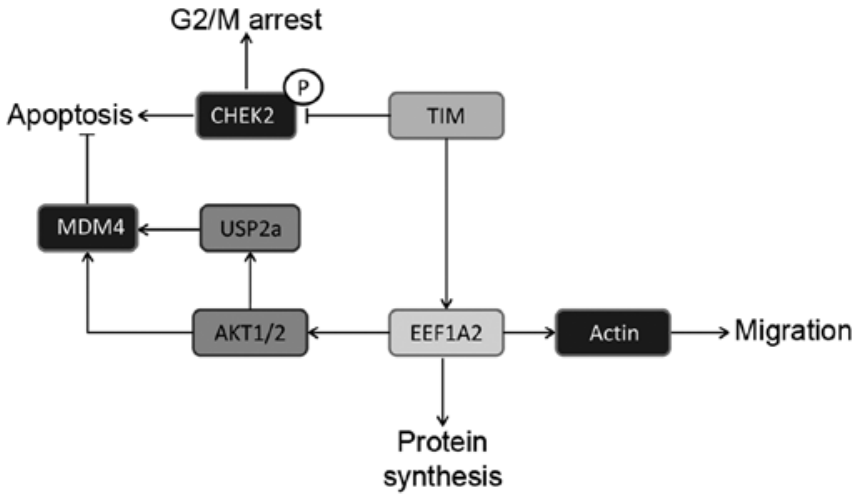

Figure 8. Schematic drawing of the protumorigenic TIM functions. TIM overexpression prevents CHEK2 phosphorylation and via EEF1A2 results in activation of PI3/AKT/mTOR-signaling that leads to stabilization of MDM4 via AKT and USP2a as described previously (21), thereby inhibiting apoptosis. In addition, EEF1A2 promotes protein biosynthesis of tumor cells and supports migration via direct interaction with actin.

AKT signaling cascade as well as promotion of tumor cell migration. A schematic model of the protumorigenic mechanisms activated by TIM overexpression is shown in Fig. 8.

\section{Discussion}

By demonstrating the epigenetic inactivation of Period homolog 3 we have recently shown that dysregulation of clock machinery components occurs in human HCC (15). We hypothesized that altered clock function may be a hallmark of hepatocarcinogenesis and found six clock genes (CLOCK, CSNK1E, PER1, PER2, RORA and TIM; Gene Expression Omnibus Series GSE50579) to be differentially expressed on the mRNA level between HCC and normal liver. We focused on TIM as it has been shown to be important for the maintenance of chromosome integrity and growth control $(5,6)$, and a recent meta-analysis revealed a transcriptional timeless-dependent network and suggested a role of TIM in development and progression of various epithelial cancer types (e.g., bladder, breast, cervical, colorectal, gastric, head and neck, kidney, lung and prostate) (26). Here, we observed that TIM is overexpressed in a subset of human HCCs, which is contradictory to a study reporting downregulation of TIM in HCC (27). Our functional analysis supports an oncogenic function as TIM knockdown resulted in reduced viability of HCC cells due to the induction of apoptosis and cell cycle arrest in vitro. The effect on proliferation may be related to the fact that TIM-TIPIN complexes have been shown to stabilize the replication fork during early $S$ phase. Depletion of TIM resulted in defects of mitotic progression, impaired sister chromatid cohesion and chromosome fragmentation (28). In line with a previous report showing that TIM is required for ATM-dependent CHEK2 activation and G2/M checkpoint control (8), we observed that siRNA-mediated inhibition of TIM in HCC cells increased CHEK2 phosphorylation resulting in cell cycle arrest. Together, these data provide evidence that upregulation of TIM in HCC cells promotes tumor cell proliferation. Although systemic targeting of TIM might exert antitumoral activity in overexpressing tumors, such an approach is probably limited by its physiological function in the replication fork (28), suggesting that the spectrum of unwanted side-effects following targeting of TIM might be similar to conventional chemotherapy due to broad unspecific targeting of any proliferating cell. Accordingly, specific targeting of the relevant 'non-canonical' mediators of the oncogenic TIM function might be a more promising therapeutic strategy.

Recent data indicated that EEF1A2 overexpression is a hallmark of prostate and ovarian cancer and plays an important role in mammary carcinogenesis (29-31). We have previously identified EEF1A2 as a candidate oncogene in human hepatocarcinogenesis (20). EEF1A2 acts as an upstream inducer of the PI3K/AKT/mTOR axis leading to functional inactivation of p53 by posttranslational stabilization of the mouse double minute homolog 4 (MDM4) in human HCCs (21).

Interaction of EEF1A with the cytoskeleton is important for efficient translation and EEF1A is involved in the organization of the actin cytoskeleton in vivo $(32,33)$.

Herein, we demonstrate that EEF1A2 directly interacts with TIM and $\beta$-actin. Since our data indicate that the TIM-EEF1A2 interaction occurs likely in the cytoplasm, we speculate that TIM exerts its oncogenic function at least partially in this compartment. In line, knockdown of TIM reduced EEF1A2 expression and impaired tumor cell migration. EEF1A2 has been shown to induce filopodia formation in cell lines and enhance migration in an AKT- and PI3K-dependent manner $(34,35)$ indicating that the reduced activation of the EEF1A2/ $\mathrm{PI} 3 \mathrm{~K} / \mathrm{AKT} / \mathrm{mTOR}$ axis may contribute to the migratory phenotype observed in this study.

In addition to its canonical function in translational elongation EEF1A exerts a plethora of non-canonical activities including export of proteins from the nucleus (36), a role in viral replication (37), and interaction with the proteasome which is increased when translation is inhibited (38). As EEF1A2 protein levels could not be rescued by pharmacological inhibition of the proteasome after TIM knockdown, the latter function is unlikely to contribute to the decreased expression of various proteins observed here. The most likely explanation is that the EEF1A2 knockdown impaired ribosomal function resulting in reduced protein biosynthesis in HCC cells as indicated by significantly decreased EEF1A2 protein levels following cycloheximide treatment.

In conclusion, TIM exerts protumorigenic functions in human HCC, which are crucially mediated by physical interaction with EEF1A2 and the activation of its downstream signaling cascade as well as promotion of tumor cell migration via EEF1A2-mediated activation of actin dynamics. Although targeting of TIM and EEF1A2 was effective in vitro, EEF1A2 seems to be the more promising target as its physiological expression level is restricted to non-proliferating cells and de novo expression is frequently found in human cancers. Future studies are needed to evaluate both the efficacy as well as potential unwanted effects of these treatment approaches in vivo.

\section{Acknowledgements}

We are grateful to Ariane Eberhardt, Veronika Geissler, Sara Messard, Verena Kautz, and Eva Eiteneuer for excellent technical assistance. This study was supported by the Deutsche 
Forschungsgemeinschaft DFG SFB/TRR77 (subproject B5 to R.P, P.S. and T.L.), the Deutsche Krebshilfe (no. 110885 to T.L. and O.N.), and the Tissue Bank of the National Center for Tumor Diseases Heidelberg. N.E. received a stipend from the Egyptian Ministry of Higher Education.

\section{References}

1. Reppert SM and Weaver DR: Coordination of circadian timing in mammals. Nature 418: 935-941, 2002.

2. Ko $\mathrm{CH}$ and Takahashi JS: Molecular components of the mammalian circadian clock. Hum Mol Genet $15 \mathrm{Spec}$ No 2: R271-R277, 2006

3. Ceriani MF, Darlington TK, Staknis D, et al: Light-dependent sequestration of TIMELESS by CRYPTOCHROME. Science 285: 553-556, 1999.

4. Benna C, Scannapieco P, Piccin A, et al: A second timeless gene in Drosophila shares greater sequence similarity with mammalian tim. Curr Biol 10: R512-R513, 2000.

5. Matsuo T, Yamaguchi S, Mitsui S, Emi A, Shimoda F and Okamura H: Control mechanism of the circadian clock for timing of cell division in vivo. Science 302: 255-259, 2003.

6. Unsal-Kacmaz K, Mullen TE, Kaufmann WK and Sancar A Coupling of human circadian and cell cycles by the timeless protein. Mol Cell Biol 25: 3109-3116, 2005.

7. Unsal-Kacmaz K, Chastain PD, Qu PP, et al: The human Tim/Tipin complex coordinates an Intra-S checkpoint response to UV that slows replication fork displacement. Mol Cell Biol 27 : 3131-3142, 2007.

8. Yang X, Wood PA and Hrushesky WJ: Mammalian TIMELESS is required for ATM-dependent CHK2 activation and G2/M checkpoint control. J Biol Chem 285: 3030-3034, 2010.

9. Barnes JW, Tischkau SA, Barnes JA, et al: Requirement of mammalian Timeless for circadian rhythmicity. Science 302: 439-442, 2003.

10. Gotter AL, Manganaro T, Weaver DR, et al: A time-less function for mouse timeless. Nat Neurosci 3: 755-756, 2000.

11. Wood LD, Parsons DW, Jones S, et al: The genomic landscapes of human breast and colorectal cancers. Science 318: 1108-1113, 2007.

12. Fu L, Pelicano H, Liu J, Huang P and Lee C: The circadian gene Period2 plays an important role in tumor suppression and DNA damage response in vivo. Cell 111: 41-50, 2002.

13. Fu A, Leaderer D, Zheng T, Hoffman AE, Stevens RG and Zhu Y: Genetic and epigenetic associations of circadian gene TIMELESS and breast cancer risk. Mol Carcinog 51: 923-929, 2012.

14. Mazzoccoli G, Panza A, Valvano MR, et al: Clock gene expression levels and relationship with clinical and pathological features in colorectal cancer patients. Chronobiol Int 28: 841-851, 2011.

15. Neumann O, Kesselmeier M, Geffers R, et al: Methylome analysis and integrative profiling of human HCCs identify novel protumorigenic factors. Hepatology 56: 1817-1827, 2012.

16. Lee MH and Surh YJ: eEF1A2 as a putative oncogene. Ann NY Acad Sci 1171: 87-93, 2009.

17. Knudsen SM, Frydenberg J, Clark BF and Leffers H: Tissuedependent variation in the expression of elongation factor-1 alpha isoforms: isolation and characterisation of a cDNA encoding a novel variant of human elongation-factor 1 alpha. Eur J Biochem 215: 549-554, 1993.

18. Lee S, Francoeur AM, Liu S and Wang E: Tissue-specific expression in mammalian brain, heart, and muscle of S1, a member of the elongation factor-1 alpha gene family. J Biol Chem 267: 24064-24068, 1992.

19. Ruest LB, Marcotte R and Wang E: Peptide elongation factor eEF1A-2/S1 expression in cultured differentiated myotubes and its protective effect against caspase-3-mediated apoptosis. J Biol Chem 277: 5418-5425, 2002.
20. Schlaeger C, Longerich T, Schiller C, et al: Etiology-dependent molecular mechanisms in human hepatocarcinogenesis. Hepatology 47: 511-520, 2008.

21. Pellegrino R, Calvisi DF, Neumann O, et al: EEF1A2 inactivates p53 by way of PI3K/AKT/mTOR-dependent stabilization of MDM4 in hepatocellular carcinoma. Hepatology 59: 1886-1899, 2014.

22. Pfaffl MW: A new mathematical model for relative quantification in real-time RT-PCR. Nucleic Acids Res 29: e45, 2001.

23. Longerich T, Breuhahn K, Odenthal M, Petmecky K and Schirmacher P: Factors of transforming growth factor beta signalling are co-regulated in human hepatocellular carcinoma. Virchows Arch 445: 589-596, 2004.

24. Straub BK, Boda J, Kuhn C, et al: A novel cell-cell junction system: the cortex adhaerens mosaic of lens fiber cells. J Cell Sci 116: 4985-4995, 2003

25. Smith KD, Fu MA and Brown EJ: Tim-Tipin dysfunction creates an indispensible reliance on the ATR-Chk1 pathway for continued DNA synthesis. J Cell Biol 187: 15-23, 2009.

26. Mao Y, Fu A, Leaderer D, Zheng T, Chen K and Zhu Y: Potential cancer-related role of circadian gene TIMELESS suggested by expression profiling and in vitro analyses. BMC Cancer 13: 498, 2013.

27. Lin YM, Chang JH, Yeh KT, et al: Disturbance of circadian gene expression in hepatocellular carcinoma. Mol Carcinog 47: 925-933, 2008

28. Leman AR, Noguchi C, Lee CY and Noguchi E: Human Timeless and Tipin stabilize replication forks and facilitate sister-chromatid cohesion. J Cell Sci 123: 660-670, 2010.

29. Scaggiante B, Dapas B, Bonin S, et al: Dissecting the expression of EEF1A1/2 genes in human prostate cancer cells: the potential of EEF1A2 as a hallmark for prostate transformation and progression. Br J Cancer 106: 166-173, 2012.

30. Pinke DE and Lee JM: The lipid kinase PI4KIIIbeta and the eEF1A2 oncogene co-operate to disrupt three-dimensional in vitro acinar morphogenesis. Exp Cell Res 317: 2503-2511, 2011.

31. Pinke DE, Kalloger SE, Francetic T, Huntsman DG and Lee JM: The prognostic significance of elongation factor eEF1A2 in ovarian cancer. Gynecol Oncol 108: 561-568, 2008.

32. Gross SR and Kinzy TG: Improper organization of the actin cytoskeleton affects protein synthesis at initiation. Mol Cell Biol 27: 1974-1989, 2007.

33. Gross SR and Kinzy TG: Translation elongation factor $1 \mathrm{~A}$ is essential for regulation of the actin cytoskeleton and cell morphology. Nat Struct Mol Biol 12: 772-778, 2005.

34. Amiri A, Noei F, Jeganathan S, Kulkarni G, Pinke DE and Lee JM: eEF1A2 activates Akt and stimulates Akt-dependent actin remodeling, invasion and migration. Oncogene 26: 3027-3040, 2007

35. Jeganathan S and Lee JM: Binding of elongation factor eEF1A2 to phosphatidylinositol 4-kinase beta stimulates lipid kinase activity and phosphatidylinositol 4-phosphate generation. J Biol Chem 282: 372-380, 2007

36. Khacho M, Mekhail K, Pilon-Larose K, Pause A, Cote J and Lee S: eEF1A is a novel component of the mammalian nuclear protein export machinery. Mol Biol Cell 19: 5296-5308, 2008.

37. Li D, Wei T, Abbott CM and Harrich D: The unexpected roles of eukaryotic translation elongation factors in RNA virus replication and pathogenesis. Microbiol Mol Biol Rev 77: 253-266, 2013.

38. Chuang SM, Chen L, Lambertson D, Anand M, Kinzy TG and Madura K: Proteasome-mediated degradation of cotranslationally damaged proteins involves translation elongation factor $1 \mathrm{~A}$. Mol Cell Biol 25: 403-413, 2005. 